\title{
Estrategias basadas en análisis de redes sociales para optimizar el clima de aprendizaje en la universidad
}

\author{
Strategies based on social network analysis for enhancing the learning climate at \\ universities
}

Estratègies amb anàlisis de xarxes socials per a millorar el clima d'aprenentatge a la universitat

\author{
Fran J. Garcia Garcia ${ }^{1, *}{ }^{\oplus}$, Evelyn E. Moctezuma-Ramírez ${ }^{1,2}{ }^{\circledR}$, Cristian \\ Molla-Esparza ${ }^{3 \odot}$, Inmaculada López-Francés ${ }^{1 \odot}$ \\ 1 | Departament de Teoria de I'Educació, Universitat de València, Valencia, España \\ 2 | Universidad Autónoma del Estado de Morelos, Morelos, México \\ 3 | Department de Mètodes d'Investigació i Diagnòstic en Educació, Universitat de València, Valencia, España \\ *Autor para correspondencia: garfran9@uv.es (Fran J. Garcia Garcia)
}

Recibido: 06/11/2020 | Aceptado: 22/02/2021 | Publicado: 19/07/2021

Cómo citar: Garcia-Garcia, F.J., Moctezuma-Ramírez, E. E. Molla-Esparza, C. y López-Francés, I. (2021). Estrategias basadas en análisis de redes sociales para optimizar el clima de aprendizaje en la universidad. Research in Education and Learning Innovation Archives, 27,33-46.

10.7203/realia.27.18960

Copyright: El/La Autor/a. Open Access: Este es un artículo de acceso abierto distribuido bajo los términos de la licencia Creative

Commons

Attribution-NoDerivatives

4.0 International licence (CC BY-ND 4.0)

Financiación: Ministerio de Educación, Cultura y Deporte, España (FPU17/00156); CONACyT, México (2020-00002602NACF-19326); Generalitat Valenciana, España (ACIF/2017/347).
RESUMEN: El objetivo de este estudio fue detectar líderes a partir de los puntajes de centralidad de un grupo de estudiantes universitarios para repartir funciones que contribuyeran a optimizar el clima del aula. Entendimos que el clima mejoraba estimulando la construcción de conocimiento en red desde un entorno de aprendizaje colaborativo con apoyo de computadoras. La experiencia educativa se llevó a cabo en un entorno virtual. Se configuró un foro de discusión en línea para los estudiantes con formato loosely-structured y se calcularon los puntajes de centralidad a partir de la red social que generaron en el foro. Los resultados muestran un crecimiento significativo en la conectividad de los estudiantes y se detectaron diferentes estilos de liderazgo, en función de los cuales diseñamos estrategias para optimizar el clima del aula de manera autorregulada y estable. Dependiendo del tipo de centralidad, se detectaron líderes en popularidad, en sociabilidad, en cercanía a los demás, en control de la información que fluye a través de la red y líderes en influencia. La novedad del estudio consiste en la producción incipiente de tecnología educativa basada en Análisis de Redes Sociales, y concretamente el diseño de estrategias basadas en la centralidad para optimizar el clima de un aula universitaria.

PALABRAS CLAVE: redes sociales; discusión en línea; tecnología educativa; liderazgo; educación superior

ABSTRACT: The aim of this study was to detect leaders among a group of university students based on their centrality scores and, from these scores, to distribute roles to help enhance learning climate. We understood that learning climate improved when the construction of networked knowledge was stimulated from the perspective of Computer-Supported Collaborative Learning. We conducted the educational experiment in a virtual environment. An online discussion forum was set up in a loosely structured format, and the students' centrality scores were calculated from the social network they generated in the forum. Our findings show that student connectivity increased significantly and that several leadership styles were detected. Based on these leadership styles we designed strategies for optimizing learning climate in a self-regulated and stable way. Based on the type of centrality, we detected leaders in terms of their popularity, sociability, closeness to others, control 
of information flowing through the network, and influence. The novelty of this study resides in the incipient production of educational technology based on Social Network Analysis and, specifically, on the design of centrality-based strategies for optimizing climate in the university classroom.

KEYWORDS: social networks; online discussion; educational technology; leadership; higher education

RESUM: L'objectiu d'aquest estudi va ser detectar líders a partir de les puntuacions de centralitat d'un grup d'estudiants universitaris per repartir funcions que contribuïren a optimitzar el clima de l'aula. Vam entendre que el clima millorava estimulant la construcció de coneixement en xarxa des d'un entorn d'aprenentatge col-laboratiu amb suport d'ordinadors. L'experiència educativa es va dur a terme en un entorn virtual. Es va configurar un fòrum de discussió en línia per als estudiants amb format loosely-structured i es van calcular les puntuacions de centralitat a partir de la xarxa social que generaren en el fòrum. Els resultats mostren un creixement significatiu en la connectivitat dels estudiants i es van detectar diferents estils de lideratge, en funció dels quals dissenyarem estratègies per a optimitzar el clima de l'aula de manera autoregulada i estable. Depenent del tipus de centralitat, es van detectar líders en popularitat, en sociabilitat, en proximitat, en control de la informació que flueix a través de la xarxa i líders en influència. La novetat de l'estudi consisteix en la producció incipient de tecnologia educativa basada en l'anàlisi de xarxes socials, i concretament el disseny d'estratègies basades en la centralitat per a millorar el clima d'una aula universitària.

PARAULES CLAU: xarxes socials; discussió en línia; tecnologia educativa; lideratge; educació superior

\section{Notas de aplicación práctica}

\section{Qué se sabe sobre el tema}

- Existen modelos basados en Análisis de Redes Sociales para detectar comunidades de aprendizaje en educación superior, que construyen conocimiento en red sobre temas específicos.

- Los foros de discusión en línea constituyen redes sociales donde los estudiantes universitarios van construyendo conocimiento en red. El conocimiento se construye más y mejor a medida que los estudiantes están más presentes en los foros.

- No se han publicado todavía suficientes evidencias empíricas en educación superior para disponer de una tecnología educativa basada en los puntajes de centralidad, que contribuya a construir conocimiento en red a partir del liderazgo de los estudiantes universitarios.

\section{Qué aporta este trabajo}

- Proponemos un método de detección de líderes en el aula con base en los puntajes de centralidad, que permite diseñar estrategias para optimizar un clima de construcción del conocimiento en red.

- La novedad de este estudio consiste en la producción de tecnología educativa basada en Análisis de Redes Sociales, aunque todavía es una tecnología en fase incipiente.

\section{Implicaciones para la práctica y/o política}

- Las estrategias basadas en la centralidad que proponemos en el artículo son viables siempre que la conectividad de los estudiantes crezca de manera significativa a lo largo del tiempo de formación.

- Es necesario obtener más evidencias empíricas acerca de la funcionalidad de estas estrategias de cara a la construcción de conocimiento en red, pero el método que proponemos permite diseñarlas.

\section{INTRODUCCIÓN}

La tecnología educativa basada en Análisis de Redes Sociales (ARS) incluye medidas de centralidad, que son útiles para detectar diferentes estilos de liderazgo en una red. Cuando esa red representa un aula universitaria, se pueden definir estrategias basadas en la centralidad de los estudiantes para optimizar el clima del aula, involucrando a 
varias personas "clave". Uno de los instrumentos más recurrentes para extraer datos y realizar un ARS son los foros de discusión en línea (Mohammed, 2005; Silva, Barbosa, y Gomes, 2019; Zou et al., 2021). De acuerdo con los últimos hallazgos, la discusión en línea favorece la participación de los estudiantes universitarios (Chen y Liu, 2020) y su construcción del conocimiento de manera colaborativa (van Heijst, de Jong, van Aalst, de Hoog, y Kirschner, 2019). Esta puede ser una estrategia útil para proyectar su participación en clase y favorecer un clima apropiado en el aula, aprovechando un conjunto de datos analizables.

El clima del aula es resultado del estado de ánimo, actitudes y normas que se comparten en clase. Nuestra idea fue procurar un clima interactivo, inclusivo y formativo, a partir del ARS. Buscamos un clima donde los estudiantes no estuvieran tan preocupados por las calificaciones, sino también por su proceso de aprendizaje.

Muchos de los estudios publicados en este sentido se fundamentan en una pedagogía constructivista y en un enfoque cognitivo-social del aprendizaje (Dommett, 2018). Por lo tanto, el propósito general de utilizar foros de discusión en línea es producir un clima que estimule la construcción de conocimiento en red. Este clima puede estabilizarse de manera autorregulada a lo largo de un cierto periodo de formación, siguiendo modelos de corregulación del aprendizaje (Hadwin, Järvelä, y Miller, 2018). De hecho, todavía se están valorando los efectos del e-learning basados en la regulación social (Hwang, Wang, y Lai, 2021) y las métricas de centralidad brindan criterios razonables para gestionar un aula universitaria y mejorar la construcción de conocimiento en red.

El objetivo de este estudio fue detectar distintos tipos de líder a partir de la centralidad de los estudiantes para asignar funciones de liderazgo que contribuyeran a optimizar el clima del aula. Partimos de la hipótesis de que el clima mejora a medida que se estimula la construcción del conocimiento en red desde un entorno de aprendizaje colaborativo con apoyo de computadoras. El estudio tuvo una orientación exploratoria, ya que se buscaron evidencias empíricas tras el pilotaje de una actividad de innovación docente. La mayor parte de estudios publicados sobre ARS aplicado a foros de discusión en la universidad pretendieron establecer un marco teórico para el propio análisis (Jan y Vlachopoulos, 2019) o se centraron en el diagnóstico de redes de aprendizaje (Jan, 2018). Sin embargo, no encontramos tantos estudios sobre técnicas educativas basadas en ARS para estimular el aprendizaje. La novedad y relevancia de nuestra experiencia radica precisamente en la producción incipiente de tecnología educativa basada en ARS.

El foro de discusión en línea no se llevó a cabo para obtener evidencias de validez y fiabilidad para ningún método didáctico. Se trató del pilotaje de una actividad de innovación docente, que todavía necesita evidencias empíricas para avalar su efectividad. Con esta idea en mente, se diseñaron estrategias de enseñanza para mejorar el clima del aula de manera autorregulada a partir de las métricas de centralidad extraídas de las publicaciones en el foro.

\section{MÉTODO}

Este artículo es un informe acerca del pilotaje de una actividad diseñada para evaluar la centralidad de un grupo de estudiantes universitarios. La actividad consistió en un foro de discusión en línea, que ofrecía la posibilidad de recabar los datos necesarios, de acuerdo con las recomendaciones publicadas en estudios anteriores (Lucas, Gunawardena, y Moreira, 2014). Además, el uso de foros es habitual en nuestro contexto (DuráMartínez, 2010) y asumimos este estudio como una contribución para el desarrollo de la docencia en las universidades de nuestra región. El foro se aplicó durante 10 semanas. 


\subsection{Participantes y contexto de aprendizaje}

La actividad se llevó a cabo en la Universitat de València con un grupo de estudiantes de primer curso, matriculados en el grado de Educación Social. Se planteó un foro de discusión virtual para evaluar la participación en la asignatura de Pedagogía Social. De acuerdo con el programa docente, la evaluación de la materia se dividía en tres bloques. Los estudiantes entregaron un portafolios con actividades teóricas que valía un $50 \%$ sobre la calificación total. El 30\% de la nota dependió de un proyecto que diseñaron en equipo y el $20 \%$, de su participación en el desarrollo de la asignatura. Sin embargo, no quedaba claro cómo medir la participación, aunque ocupaba un alto porcentaje. En ese momento pensamos que los estudiantes debían participar más allá de la asistencia a clase y las intervenciones en el aula, de modo que configuramos el foro para participar $\mathrm{y}$ aprender en red de manera asíncrona.

Este grupo estaba compuesto por 50 estudiantes, entre los cuales había 11 varones y 39 mujeres. La mortalidad experimental durante el pilotaje no fue motivo para cancelar el ensayo, aunque al principio barajábamos la posibilidad de una participación irregular que no nos permitiera alcanzar conclusiones firmes sobre los objetivos iniciales. Una vez finalizada la actividad, tuvimos que excluir a tres alumnas de la muestra porque su participación había sido demasiado escasa y distorsionaba el análisis de resultados. La muestra que analizamos finalmente incluyó 47 estudiantes y también al profesor, ya que participó en la actividad con la publicación de una primera entrada en el foro. Esta entrada sirvió como ejemplo y abrió un tema de debate, ya que se preguntó a los estudiantes si consideraban útil esta asignatura y por qué.

La tarea principal consistió en leer los comentarios publicados en el foro y contestarlos, de forma que se estableciera un diálogo entre iguales para construir y "negociar" (Fabbri, 2018) el conocimiento en red. En este sentido, el foro constituyó una red de aprendizaje dirigida, donde un estudiante interpelaba a otro a propósito de sus comentarios, sin que éste diera una respuesta o interactuase necesariamente con el primero.

\subsection{Objetivos de la tarea}

La actividad tuvo tres objetivos fundamentales: (1) aprender sobre pedagogía social y (2) acerca de los compañeros, y (3) mejorar la experiencia formativa del grupo. Estos objetivos se concretaron de la siguiente manera, respectivamente.

- Que los estudiantes construyeran su propio conocimiento relacionado con la pedagogía social de manera asíncrona, al mismo tiempo que interactuaban entre sí en un entorno común de aprendizaje en línea. Entendimos la interacción como un factor necesario para construir conocimiento en red.

- Que conocieran mejor los intereses cognitivos y profesionales del resto de sus compañeros, estableciendo lazos con el mayor número de personas posible.

- Que el profesor dispusiera de métricas de centralidad para mejorar el clima del aula, optimizando así la experiencia formativa de los estudiantes.

Nuestro estudio se centra fundamentalmente en el tercer objetivo, ya que es el único que no pudimos contrastar con otras actividades de la asignatura que ofrecieran resultados de aprendizaje similares. El propósito de obtener métricas de centralidad se concretó en cinco objetivos operativos: detectar líderes en popularidad, en sociabilidad, en influencia, en acceso a los compañeros y en regulación de los flujos de información en el foro. Los estilos de liderazgo vienen explicados en más detalle en los apartados 2.5 y 3.1. La detección de líderes facilitó la producción de estrategias docentes para mejorar el clima del aula y favorecer la autorregulación. 


\subsection{Instrumentos y configuración del entorno virtual}

En la Universitat de València trabajamos habitualmente con la plataforma Moodle (htt ps://moodle.org/). Disponemos de un aula virtual ordenada por asignaturas y cursos, en torno a la cual gestionamos nuestra actividad docente. Por tanto, configuramos el foro en esta plataforma que nos facilitó la propia institución.

Durante la configuración, buscamos un título sugerente para atraer la participación de los estudiantes, añadimos una descripción breve sobre el sentido de la actividad y no restringimos el acceso de los estudiantes al foro en ningún momento. Nos decantamos por un formato loosely-structured (Dommett, 2018), aunque algo más estructurado de lo que se prevé en las clasificaciones tradicionales sobre los tipos de configuración posibles para organizar un foro (Hammond, 2005). Más adelante indicamos las normas de participación que definen la estructura que diseñamos para la actividad.

Cuando configuramos el foro, pensamos que sería buena idea que los estudiantes pudieran cargar archivos en sus entradas. Esto les permitiría aportar pruebas sobre sus argumentos, materiales para ampliar información u otros recursos similares. Sin embargo, nadie cargó archivos. Algunos estudiantes añadieron archivos de imagen directamente en su publicación, junto con el texto, y otros incluyeron la dirección URL a páginas web con información complementaria. De todas formas, habilitamos la opción de cargar archivos y la mantuvimos habilitada durante todo el curso.

Empleamos el sistema de reconocimiento de texto Urkund (https://www.urkund.co $\mathrm{m} /$ ), que funciona de manera automática y sirve para detectar la cantidad de plagio en cada entrada de forma individual. Un plagio superior al $15 \%$ se penalizó con una reducción sobre la calificación correspondiente a esta parte de la evaluación en la asignatura. La penalización fue progresiva. Cuanto mayor era el plagio, mayor era la penalización. Este fue el único sistema relacionado con la evaluación que configuramos en el foro. No utilizamos ningún sistema de la plataforma para calificar a los estudiantes, ya que eran puntajes basados en reducciones, como la que se aplicó en función del plagio, y era más sencillo evaluarlos utilizando una hoja de cálculo de Microsoft Excel.

Las conexiones entre los estudiantes se volcaron directamente en un software para el ARS, a fin de tener en cuenta otros aspectos de la evaluación. En este caso utilizamos la versión 0.9.2 de Gephi (https://gephi.org/), ya que las prestaciones de Moodle no incluyen la posibilidad de evaluar a los estudiantes con parámetros basados en ARS. Podríamos haber instalado algunos plug-in, pero los que había disponibles no ofrecían las prestaciones que necesitábamos para calcular la centralidad de los estudiantes.

\subsection{Normas de participación en red}

En el momento de diseñar la actividad establecimos algunas normas de participación en el foro. El propósito de aplicar estas normas fue principalmente lograr los objetivos previstos al inicio, pero también facilitar la evaluación para el profesor y el manejo de la plataforma para los estudiantes. Se procuró reducir la cantidad de normas en la medida de lo posible y se asignó un nombre a cada norma para memorizarlas mejor. Finalmente se establecieron las siguientes normas.

- Título: se puso título a cada entrada para identificar rápidamente su contenido.

- $\mathrm{P} / \mathrm{R}$ : las entradas fueron identificadas como preguntas o respuestas, pero nunca como las dos al mismo tiempo.

- Dirección: cada entrada estuvo referida a otras entradas publicadas previamente en el foro para preguntar o responder acerca de su contenido.

- Mínimos: se publicó al menos una entrada cada semana hasta que finalizaron las clases de esta asignatura. 
- Evaluación: se indicó al pie de la entrada la utilidad de cada referencia en una escala del 1 al 3, que permitió asignar pesos a las aristas en la red.

- No-cambios: no se pudieron modificar las entradas, una vez publicadas en el foro.

De acuerdo con algunas investigaciones, cuando los estudiantes tienen la posibilidad de encontrar y seleccionar la información que necesitan, y sobre todo cuando disponen de avisos o recomendaciones en el foro, suelen leer y contestar a sus compañeros con más frecuencia (P.-Y. Wang y Yang, 2012). Nosotros no activamos avisos porque podrían reducir la carga cognitiva que son capaces de soportar los estudiantes (Sachdeva y Gilbert, 2020), entorpeciendo su capacidad de atención y favoreciendo un clima apático en el grupo. En su lugar, les recomendamos utilizar el buscador para filtrar los comentarios publicados.

En el caso de Moodle, el buscador no incluía los títulos de las entradas en el motor de búsqueda, de modo que recomendamos también que utilizasen las palabras del título en el cuerpo del texto de sus entradas y que aportaran títulos breves e informativos. Entendemos que esto les ayudó a encontrar la información que necesitaban en cada momento y pudieron canalizar la actividad hacia sus intereses de formación.

Cada estudiante pudo saber si la información que proporcionaba en el foro era de utilidad para el grupo, ya que los compañeros la iban valorando cuando publicaban. Con todo, la evaluación entre pares no se tomó en cuenta para las calificaciones en ninguna circunstancia y así se clarificó en el aula varias veces desde el principio.

Aunque el propósito no fue detectar preguntas frecuentes (FAQ), nos inspiramos en el modelo question answering forum (Sindhgatta, Marvaniya, Dhamecha, y Sengupta, 2017) para la norma $P / R$, puesto que las preguntas favorecen la construcción del conocimiento (Gargallo-López, 2017). Por eso organizamos una red de aprendizaje en la que los estudiantes dirigieran preguntas unos a otros.

La decisión de prohibir la modificación de las entradas después de su publicación respondió a la necesidad de evaluar el desarrollo de cada estudiante, tomando como referencia el tiempo de publicación de cada comentario.

Aunque al principio costó que los estudiantes siguieran todas las normas, no tardaron en aprenderlas y respetarlas.

\subsection{Evaluación de la centralidad}

En el marco del ARS, la centralidad de un nodo se define como su importancia relativa a una red de la cual ese nodo es parte integrante. El ARS ofrece un marco válido para analizar el aprendizaje y la participación en la universidad (Jan y Vlachopoulos, 2019), sobre todo cuando se trata de que los estudiantes indaguen en red acerca de nuevos temas de conocimiento (Jan, 2018). En este sentido, los nodos de la red representaron a los estudiantes. Cuando indagaron unos con respecto a la información que aportaron los otros estudiantes en el foro, se fue constituyendo una red donde fue posible identificar hasta qué punto una persona tuvo más influencia que otra, o la autoridad que adquirió dentro de esa red. Obtuvimos la centralidad de grado, la centralidad de vector propio, la cercanía y la intermediación de cada estudiante para determinar su importancia en el foro de discusión en línea.

La forma más sencilla de obtener la centralidad fue sumar el número de enlaces que tuvo un nodo $\mathrm{v} \in \mathrm{V}$ (Sun y Tang, 2011), siendo $\mathrm{v}$ el nodo y $\mathrm{V}$ el conjunto de nodos que componía la red. Estos enlaces eran los grados que tuvo cada nodo en cuestión. En nuestra red dirigida había grados de entrada, cuando el nodo era el vértice final del enlace o arista, y grados de salida, cuando el nodo era el vértice inicial. Calculamos la centralidad a partir de los grados de entrada para obtener un puntaje de popularidad y, a partir de los grados de salida, para conocer la sociabilidad de los estudiantes en el contexto del foro. Un ejemplo al margen del foro puede ayudar a entender mejor esta idea. 
Considerando una red dirigida como en el ejemplo de la Figura 1 , el nodo con mayor centralidad de grado es $\mathrm{v}_{1}$ porque tiene más enlaces $\mathrm{e}_{i}$ que el resto, pero el más popular es $\mathrm{v}_{4}$ porque tiene el doble de grados de entrada que cualquier otro nodo de la red. El nodo $\mathrm{v}_{1}$ es también el más sociable, puesto que ha producido el triple de grados de salida que cualquier otro nodo, mientras que $\mathrm{v}_{4}$ está entre los menos sociables, pese a ser el más popular.

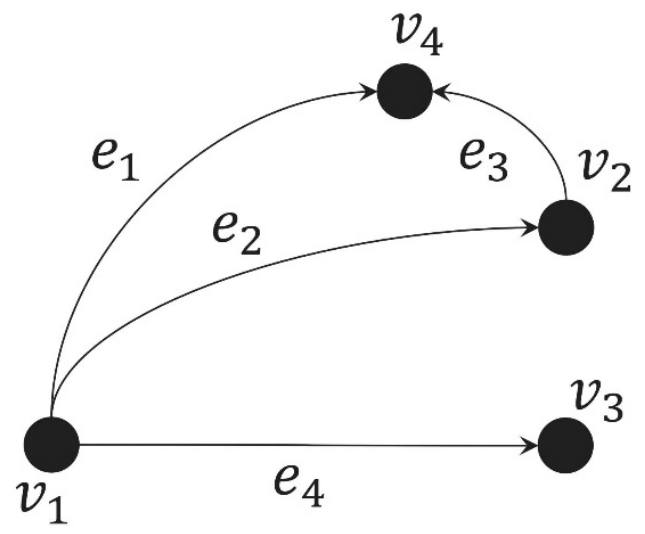

Figura 1. Ejemplo de una red dirigida I. Fuente: elaboración propia

Obtuvimos la centralidad de vector propio para conocer la influencia de cada persona en el foro de discusión. Primero calculamos el producto de Ax, donde A es la matriz de adyacencia y $\mathrm{x}$ es el vector que resulta de las centralidades de grado de los nodos que estaban conectados directamente a un nodo en cuestión. De este modo, la suma de estas centralidades dio un valor para cada nodo, que sirvió de base para contrastar la centralidad de vector propio reportada por Gephi. Los nodos con mayor centralidad de vector propio estuvieron conectados a otros nodos que también tuvieron un alto nivel de conexión, vinculando varios grupos de estudiantes, que al mismo tiempo formaban conglomerados en la red.

Se calculó la cercanía de los estudiantes, es decir, la distancia más corta en la red desde cada uno de ellos hacia todos los demás (Freeman, 1979), basándonos en el trabajo clásico de Sabidussi (1966). Esta medida aportó información sobre su accesibilidad al resto de los compañeros en las discusiones del foro. La función de cálculo consistió en sumar los vectores de la matriz de distancias i,j, considerando la distancia más corta entre el nodo i y el nodo j. Por tanto, la centralidad para cada nodo i se obtuvo calculando el valor recíproco de la suma de la matriz de distancias, cuyo iésimo componente es igual a 1 y los demás son igual a 0 . Finalmente, normalizamos la cercanía en el rango 0,1. Conocer la centralidad de los estudiantes nos permitió saber quiénes estaban más próximos al centro de la red y, por tanto, quiénes agilizaban la propagación de la información entre el resto de sus compañeros.

En el ejemplo de la Figura 2, el nodo $\mathrm{v}_{1}$ tiene una centralidad mayor que el resto y es más fácil que transmita información a toda la red, ya que sólo hay un grado de separación con los demás nodos. En cambio, los otros nodos tienen que dar el doble de pasos para propagar una información por toda la red, ya que tienen que pasar por el nodo $\mathrm{v}_{1} \mathrm{y}$ dirigirse después hacia un tercer nodo.

La intermediación permitió detectar estudiantes que estaban sirviendo de puente entre otros estudiantes en el foro, como se muestra en el ejemplo de la Figura 2 . El nodo v1 también es un puente entre todos los demás y actúa como un intermediario entre ellos. Por tanto, es un punto de control de la información que se transmite entre el resto de los nodos, de los cuales es intermediario. Calculando la intermediación conocimos la frecuencia con que un estudiante sirvió de puente a lo largo del camino 


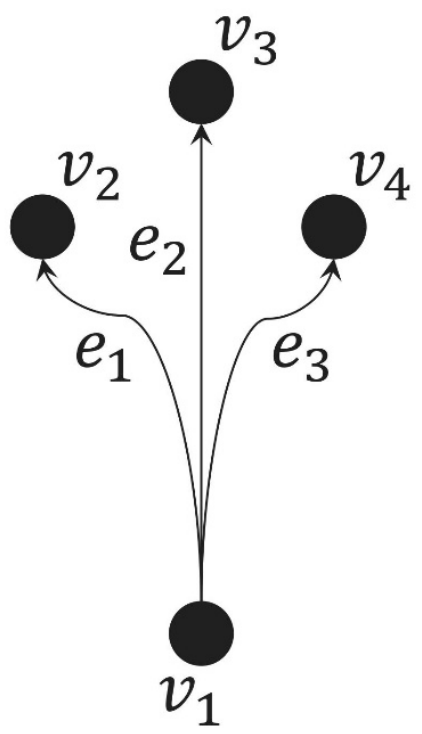

Figura 2. Ejemplo de una red dirigida II. Fuente: elaboración propia

más corto entre otros dos estudiantes, siguiendo la idea de (Freeman, 1977). Los puntajes de intermediación fueron normalizados, al igual que los de cercanía, y se utilizó el algoritmo de (Brandes, 2001) para obtener los resultados.

Cada una de estas medidas de centralidad permitió detectar diferentes tipos de líder, como se muestra en la Figura 3. Cuando llevamos a cabo el pilotaje de esta actividad, teníamos la expectativa de hallar un estilo distinto de liderazgo en diferentes personas, ya que esto permitiría asignar roles a los líderes y repartir el esfuerzo para mejorar el clima del aula.

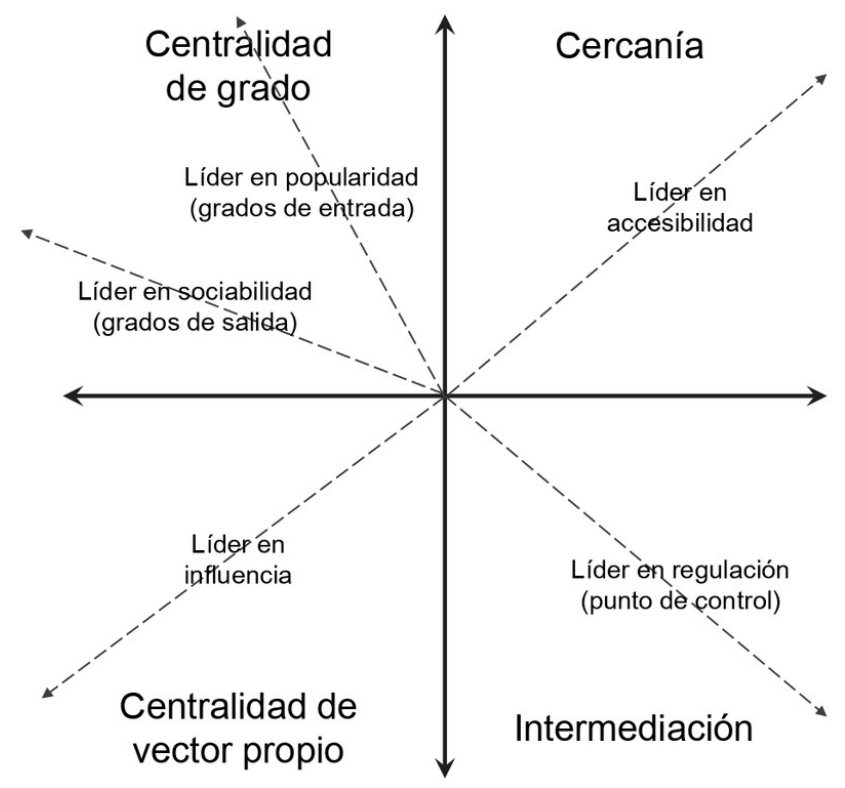

Figura 3. Estilos de liderazgo en función de la centralidad. Fuente: elaboración propia 


\section{RESULTADOS}

El crecimiento de publicaciones en el foro fue incrementándose de manera constante a lo largo de las 10 semanas de formación (Figura 4). Cada entrada generó una o más conexiones entre los estudiantes, aunque no publicaron mucho más de lo que se les pidió al inicio en las normas de la actividad.

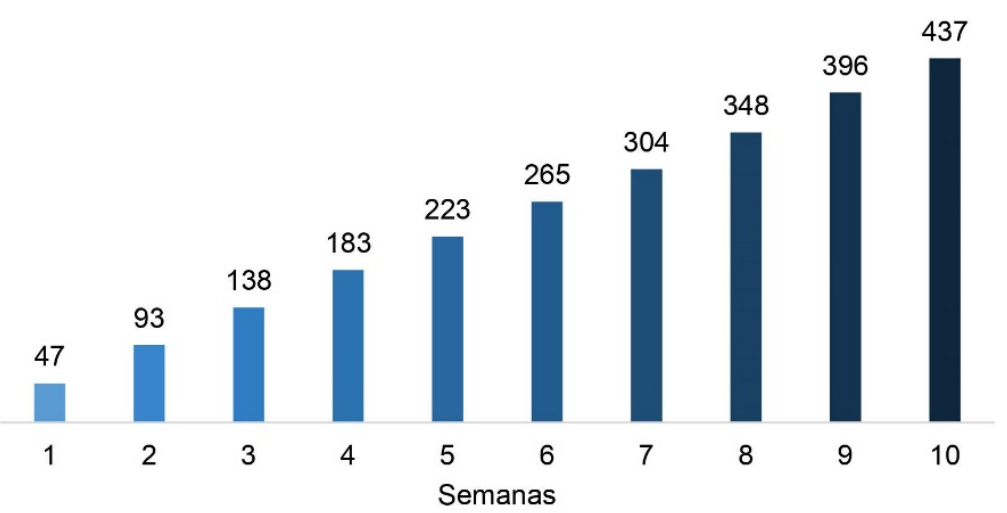

Figura 4. Crecimiento acumulado de entradas por semana. Fuente: elaboración propia

En consecuencia, la red de aprendizaje fue creciendo hasta alcanzar una densidad igual a .235 (Figura 5) y la evolución del grado de conexión entre los estudiantes fue significativa semana tras semana hasta completar las 10 semanas de formación (Tabla 1).
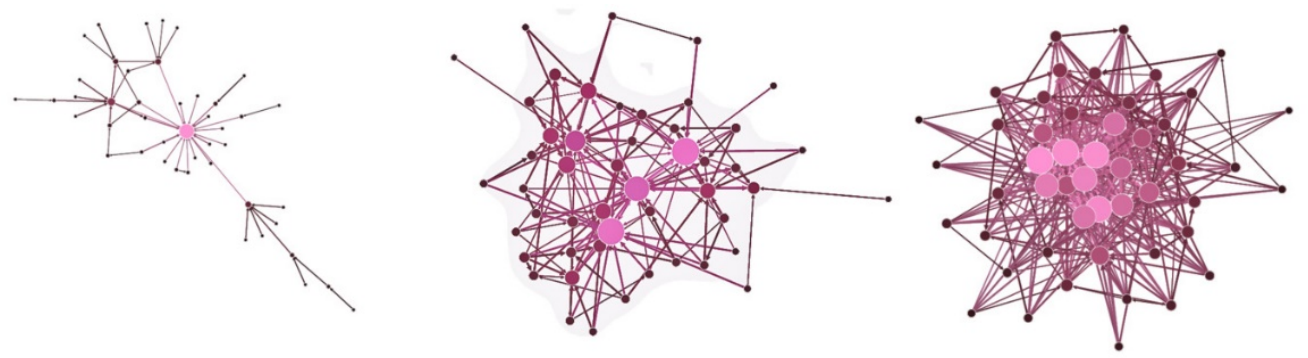

Figura 5. Evolución de la red (semanas 1-3-9). Fuente: elaboración propia

Tabla 1. Evolución del grado de conexión. Fuente: elaboración propia.

\begin{tabular}{|c|c|c|c|c|c|c|c|c|c|c|}
\hline Semana (S) & S1 & S2 & s3 & S4 & S5 & S6 & S7 & S8 & s9 & S10 \\
\hline$N$ & 49 & 49 & 49 & 49 & 49 & 49 & 49 & 49 & 49 & 49 \\
\hline Media & 2.12 & 4.33 & 7.02 & 9.02 & 10.61 & 12.73 & 14.61 & 17.10 & 19.84 & 21.92 \\
\hline Mediana & 1.00 & 3.00 & 5.00 & 6.00 & 8.00 & 10.00 & 11.00 & 13.00 & 15.00 & 16.00 \\
\hline RIC* & 1.00 & 2.00 & 5.00 & 7.00 & 8.00 & 10.00 & 11.50 & 16.50 & 19.50 & 21.50 \\
\hline Contraste & S1-10 & $\mathrm{S} 2-10$ & S3-10 & S4-10 & S5-10 & S6-10 & S7-10 & S8-10 & s9-10 & \\
\hline Z** & -6.095 & -6.094 & -6.095 & -6.096 & -6.099 & -6.100 & -6.104 & -6.113 & -5.749 & \\
\hline $\begin{array}{l}\text { Significación } \\
\text { bilateral }\end{array}$ & .000 & .000 & .000 & .000 & .000 & .000 & .000 & .000 & .000 & \\
\hline
\end{tabular}

* Rango intercuartílico

** Prueba de Wilcoxon, basado en rangos negativos 
Finalmente, logramos detectar diferentes tipos de líderes, a quienes pudimos asignar distintos roles para optimizar el clima del aula. La Tabla 2 muestra a los 10 estudiantes con mayor puntaje de centralidad después de la última semana. El estudiante 33 fue el único que acumuló dos tipos de liderazgo distintos.

Tabla 2. Detección de líderes en el aula. Fuente: elaboración propia.

\begin{tabular}{lllllll}
\hline Participantes & $\begin{array}{l}\text { Grado de } \\
\text { entrada* }\end{array}$ & $\begin{array}{l}\text { Grado de } \\
\text { salida* }\end{array}$ & Cercanía & Intermediación & CVP** & $\begin{array}{l}\text { Estilo de } \\
\text { liderazgo }\end{array}$ \\
\hline Estudiante 3 & 85 & 43 & 0.58 & 0.08 & 0.68 & Acceso \\
Estudiante 8 & 94 & 34 & 0.51 & 0.04 & 0.62 & \\
Estudiante 13 & 74 & 61 & 0.56 & 0.06 & 0.57 & Sociabilidad \\
Estudiante 33 & 129 & 25 & 0.47 & 0.09 & 0.72 & Popular/Control \\
Estudiante 34 & 18 & 26 & 0.51 & 0.06 & 0.22 & \\
Estudiante 40 & 70 & 43 & 0.55 & 0.04 & 0.51 & \\
Estudiante 41 & 80 & 26 & 0.46 & 0.01 & 0.77 & Influencia \\
Estudiante 47 & 79 & 54 & 0.55 & 0.05 & 0.77 & Influencia \\
Estudiante 49 & 79 & 42 & 0.53 & 0.05 & 0.57 & \\
Estudiante 50 & 1 & 36 & 0.55 & 0.02 & 0.02 & \\
Profesor 51 & 82 & 0 & 0.00 & 0.00 & 1.00 & \\
\hline
\end{tabular}

* Con pesos

** Centralidad de vector propio

\subsection{Estrategias basadas en la centralidad}

Desde el principio y a medida que fueron transcurriendo las semanas de formación, se fueron detectando distintos tipos de líderes en el aula. A partir de la tercera semana ya fue posible comenzar a asignarles funciones y orientar su actividad con el resto de los compañeros. Por lo general, se eligió un solo líder para cada estilo de liderazgo, que siempre estuvo determinado por los puntajes de centralidad de los estudiantes. En el transcurso de las semanas, el foro iba desarrollándose y en algunos casos se sustituyó a un determinado líder, ya que otro estudiante había adquirido un puntaje mayor en el tipo de centralidad que le otorgaba ese estilo de liderazgo.

El líder en popularidad actuó como un modelo a imitar por el resto de sus compañeros. Se le citó a una tutoría y se le explicó la técnica del modelado con base en la teoría cognitivo-social de Bandura (1986), que contrasta con las tesis conductistas. La idea era que este estudiante comprendiese la diferencia entre el aprendizaje por imitación y el condicionamiento de la conducta, de forma que pudiera verse a sí mismo como un estímulo de aprendizaje para los demás, en paralelo a la influencia de las calificaciones finales. El propósito de este estudiante en el grupo era favorecer las motivaciones internas del resto para aprender, contribuyendo a un clima del aula más formativo y no tan centrado en los resultados de la evaluación.

Una de las características del líder en sociabilidad era emitir información con más frecuencia que el resto de los estudiantes. La función de este líder fue activar a otras personas menos activas en la red, respondiendo a sus entradas y ayudando a que tuvieran más presencia en el foro. A este líder también se le citó para una tutoría, pero no se le dio ningún tipo de formación. Únicamente recibió indicaciones con el propósito de activar a determinadas personas en la red y contribuir a un clima del aula más interactivo.

Aunque hubo un líder en popularidad, con quien contactaba una cantidad importante de personas de la clase, no todos los estudiantes aprendían en base a un modelo de imitación. En muchas ocasiones, este fue el caso de los estudiantes situados en la 
periferia de la red, que tenían pocas conexiones en comparación con los demás. La función del líder en acceso a la red consistió en aumentar el nivel de conexión de los estudiantes menos conectados. Este estudiante también recibió indicaciones del profesor con el propósito de contribuir a un clima del aula más inclusivo.

El líder en control establecía puentes entre varios pares de estudiantes, que no tenían contacto directo entre sí. Su función natural era la de un intermediario, pero tenía la función potencial de producir un contacto directo entre esos pares de nodos y fomentar así la transitividad en la red. Por tanto, se le citó a una tutoría y se le explicó el concepto de transitividad. Se le dio un listado de estudiantes a los que podía interpelar en sus intervenciones en el foro, e incluso preguntar qué pensaban el uno de lo que decía el otro. De esta forma, el líder en control contribuyó a generar un clima del aula donde la información fluyera más deprisa entre estudiantes, hubiera más construcción de conocimiento en equipo y el grupo estuviera más cohesionado.

El líder en influencia también favoreció la cohesión del grupo. A diferencia del líder en control, éste servía de puente entre pares de conglomerados, y no tanto entre pares de estudiantes. Su propósito fue propagar información relevante entre los líderes de los conglomerados para optimizar la construcción de conocimiento en equipo y generar un clima del aula con mayor nivel de actualización en cuanto a las novedades y cambios de la asignatura.

Los roles de liderazgo fueron rotativos durante las semanas de formación e intentamos que varios estudiantes aprendieran a liderar en diferentes sentidos, siempre en función de sus puntajes de centralidad. Cuando otro estudiante superó el puntaje de centralidad de uno de los líderes, se le agradeció a ese líder su colaboración, se le recordó que había realizado una tarea valiosa para toda la clase y se le pidió que dejara de ejercer esta función para que otras personas pudieran aprender a ejercerla también.

\section{DISCUSIÓN Y CONCLUSIONES}

\subsection{Autorregulación del clima del aula}

El foro de discusión en línea permitió complementar modelos de corregulación del aprendizaje (Hadwin et al., 2018) pensados generalmente para grupos más pequeños de estudiantes (Järvelä, Järvenoja, Malmberg, y Hadwin, 2013), de los que todavía no se han obtenido evidencias empíricas basadas en puntajes de centralidad para determinar el rol de los estudiantes que trabajan en un mismo equipo. Después del pilotaje de esta actividad, concluimos que las estrategias basadas en la centralidad podrían resultar útiles para optimizar el clima del aula, generando una construcción de conocimiento en red coherente y autorregulada. Por tanto, consideramos que se trata de un complemento interesante para otras actividades que planteamos habitualmente, como los proyectos en equipos con un número de estudiantes relativamente pequeño.

Los puntajes de centralidad ofrecieron criterios al profesor para seleccionar a varios estudiantes delegados, que ejercieron diferentes funciones de optimización en el grupo. El reparto y la rotación de los roles de liderazgo facilitó un clima dinámico en el aula para la gestión y construcción del conocimiento en red, no sólo a nivel virtual, sino también presencial. Al menos, los resultados del pilotaje así lo sugieren en cuanto a la conectividad de los estudiantes (Tabla 1) y a su capital de liderazgo (Tabla 2).

\subsection{Otras experiencias de innovación educativa}

Otras experiencias similares se centraron en las calificaciones de los estudiantes, dando más peso al resultado que al proceso de aprendizaje (Alzahrani, 2017). Nuestro interés fue más bien el proceso y el clima que se proyectaba en el aula durante el transcurso de la asignatura. Algunos estudios han contribuido a la clasificación y modelización de los temas de discusión en el foro (Zhao, Jiang, y Gray, 2019), y al análisis del discurso 
y el aprendizaje que surgen de la interacción entre los estudiantes (Onyema, Deborah, Alsayed, Naveed, y Sanober, 2019; Z. Wang et al., 2019). Este tipo de evaluaciones tienen una rentabilidad formativa, pero están más orientadas al análisis de habilidades cognitivas de orden superior. El liderazgo que detectamos en nuestra experiencia está más enfocado en las dinámicas sociales, que producen una mejora en el aprendizaje.

Se han publicado experiencias docentes donde se utilizaron foros de discusión en línea para formar a los estudiantes en habilidades de liderazgo (Bleich, 2020), pero no se han aprovechado los beneficios del ARS para evaluar el liderazgo, como hicimos nosotros. En otras ocasiones se ha aplicado el ARS para valorar el resultado de los foros en cursos masivos (MOOC) (Amastini, Sari-Kaunang, Nefiratika, Sensuse, y Lusa, 2020; Zou et al., 2021). Nuestro estudio consistió en un ensayo piloto con estudiantes de grado y las implicaciones educativas podrían ser diferentes, debido al nivel de conocimientos que suele haber en uno y otro contexto. Generalmente, los cursos masivos son para ampliar contenidos de aprendizaje, mientras que el primer curso de un grado universitario está pensado para iniciarse en contenidos básicos de una disciplina académica.

Todavía no hemos encontrado más experiencias docentes donde se aplique el ARS a foros de discusión en línea para detectar estilos de liderazgo y orientar a los estudiantes hacia la mejora del clima del aula.

\subsection{Limitaciones e investigación emergente}

Aunque logramos los objetivos esperados, encontramos algunas limitaciones en la aplicación del foro. Los estudiantes no siempre siguieron las normas de participación, lo que aumentó el riesgo de cometer errores sistemáticos en el análisis de resultados. Además, el foro de discusión refleja solamente una parte del clima del aula, de modo que los hallazgos ofrecen una perspectiva incompleta sobre lo que sucedió realmente en clase. Aun así, el foro generó conexiones entre las personas de la clase, que probablemente continuaron conectándose al margen de esta actividad. Al menos, en el foro ocurrieron experiencias de contacto y se produjo un mayor conocimiento del resto de los compañeros del aula. En cambio, en los trabajos en equipo tradicionales sólo interactúa un número limitado de personas de la clase con una estructura de varias redes cerradas.

Somos conscientes de que este es un procedimiento complejo y que no todos los profesores universitarios tienen la formación necesaria en ARS y manejo de softwares para calcular los puntajes de centralidad. Sin embargo, esto no impide la exploración de estrategias basadas en la centralidad. Al contrario, las evidencias de este pilotaje son motivo para encontrar los recursos que faciliten a otros profesores el uso de estas estrategias docentes. Mientras tanto, convendría ampliar las evidencias empíricas al respecto para afianzar el conocimiento sobre la efectividad de estas estrategias y perfilar su utilidad en diferentes escenarios.

\section{REFERENCIAS}

Alzahrani, M. G. (2017). The effect of using online discussion forums on students' learning. Turkish Online fournal of Educational Technology, 16(1), 164-176.

Amastini, F., Sari-Kaunang, C. P., Nefiratika, A., Sensuse, D. I., y Lusa, S. (2020). Collaborative Learning in Virtual Learning Environment using Social Network Analysis: Case study Universitas Terbuka. 2020 7th International Conference on Electrical Engineering, Computer Sciences, and Informatics (EECSI), 262-269. https://doi.org/10.23919/ EECSI50503.2020.9251904

Bandura, A. (1986). Social Foundations of thought and action: A social cognitive theory. Englewood Cliffs, NJ: Prentice-Hall. 
Bleich, M. R. (2020). The Discussion Board in Online Learning: Leadership Development Opportunities. The fournal of Continuing Education in Nursing, 51(12), 541-543. https:// doi.org/10.3928/00220124-20201113-03

Brandes, U. (2001). A faster algorithm for betweenness centrality. The fournal of Mathematical Sociology, 25(2), 163-177. https://doi.org/10.1080/0022250X.2001.9990249

Chen, L. T., y Liu, L. (2020). Social Presence in Multidimensional Online Discussion: The Roles of Group Size and Requirements for Discussions. Computers in the Schools, 37(2), 116-140. https://doi.org/10.1080/07380569.2020.1756648

Dommett, E. J. (2018). Understanding student use of Twitter and online forums in higher education. Education and Information Technologies, 24, 325-343. https://dx.doi.org/ 10.1007/s10639-018-9776-5

Durá-Martínez, E. (2010). Una experiencia docente: la introducción del foro en la asignatura de Informática II en Biblioteconomía y Documentación. @tic revista d'innovació educativa, 4, 72-76. https://doi.org/10.7203/attic.4.183

Fabbri, M. (2018). Forums as a tool for negotiating knowledge in Higher Education. Research on Education and Media, 10(1), 9-19. https://doi.org/http://dx.doi.org/10.1515/rem-2018 $-0003$

Freeman, L. C. (1977). A Set of Measures of Centrality Based on Betweenness. Sociometry, 40(1), 35-41. https://doi.org/10.2307/3033543

Freeman, L. C. (1979). Centrality in networks: I. Conceptual clarification. Social Networks, 1, 215-239. https://doi.org/10.1016/0378-8733(78)90021-7

Gargallo-López, B. (2017). Enseñanza centrada en el aprendizaje y diseño por competencias en la universidad. Fundamentación, procedimientos y evidencias de aplicación e investigación. Valencia, España: Tirant lo Blanc.

Hadwin, A., Järvelä, S., y Miller, M. (2018). Self-regulation, co-regulation, and shared regulation in collaborative learning environments. En D.-H. Schunk y J.-A. Greene (Eds.), Educational psychology handbook series. Handbook of self-regulation of learning and performance (pp. 83-106). Nueva York, NY: Routledge/Taylor \& Francis.

Hammond, M. (2005). A review of recent papers on online discussion in teaching and learning in higher education. Journal of Asynchronous Learning Networks, 9(3), 9-23. http://dx.doi.org/10.24059/olj.v9i3.1782

Hwang, G. J., Wang, S. Y., y Lai, C. L. (2021). Effects of a social regulation-based online learning approach on students' learning achievements and behaviors in mathematics. Computers and Education, 160,1-19. https://doi.org/10.1016/j.compedu.2020.104031

Jan, S. K. (2018). Identifying online communities of inquiry in higher education using social network analysis. Research in Learning Technology, 26, 1-13. https://doi.org/10.25304/ rlt.v26.2064

Jan, S. K., y Vlachopoulos, P. (2019). Social Network Analysis: A Framework for Identifying Communities in Higher Education Online Learning. Technology, Knowledge and Learning, 24(4), 621-639. https://doi.org/10.1007/s10758-018-9375-y

Järvelä, S., Järvenoja, H., Malmberg, J., y Hadwin, A. F. (2013). Exploring Socially Shared Regulation in the Context of Collaboration. Fournal of Cognitive Education and Psychology, 12, 267-286.

Lucas, M., Gunawardena, C., y Moreira, A. (2014). Assessing social construction of knowledge online: A critique of the interaction analysis model. Computers in Human Behavior, 30, 574-582. https://doi.org/10.1016/j.chb.2013.07.050

Mohammed, M. (2005). A review of recent papers on online discussion in teaching and learning in higher education. Journal of Asynchronous Learning Networks, 9(3), 9-23. https://doi.org/10.24059/olj.v9i3.1782

Onyema, E. M., Deborah, E. C., Alsayed, A. O., Naveed, Q. N., y Sanober, S. (2019). Online Discussion Forum as a Tool for Interactive Learning and Communication. International Journal of Recent Technology and Engineering, 8(4), 4852-4859. https://doi.org/10.35940/ ijrte.D8062.118419 
Sabidussi, G. (1966). The centrality index of a graph. Psychometrika, 31(4), 581-603. https:// doi.org/10.1007/BF02289527

Sachdeva, C., y Gilbert, S. J. (2020). Excessive use of reminders: Metacognition and effortminimisation in cognitive offloading. Consciousness and Cognition, 85, 1-14. https:// doi.org/10.1016/j.concog.2020.103024

Silva, L. F. C. D., Barbosa, M. W., y Gomes, R. R. (2019). Measuring Participation in Distance Education Online Discussion Forums Using Social Network Analysis. Fournal of the Association for Information Science and Technology, 70(2), 140-150. https://doi.org/ 10.1002/asi.24080

Sindhgatta, R., Marvaniya, S., Dhamecha, T. I., y Sengupta, B. (2017). Inferring frequently asked questions from student question answering forums. Proceedings of the 10th International Conference on Educational Data Mining, 2017, 256-261.

Sun, J., y Tang, J. (2011). A survey of models and algorithms for social influence analysis. Social Network Data Analytics, 177-214. https://doi.org/10.1007/978-1-4419-8462-3_7

van Heijst, H., de Jong, F.-P.-C.-M., van Aalst, J., de Hoog, N., y Kirschner, P. A. (2019). Socio-cognitive openness in online knowledge building discourse: does openness keep conversations going? International fournal of Computer-Supported Collaborative Learning, 14, 165-184. https://doi.org/10.1007/s11412-019-09303-4

Wang, P.-Y., y Yang, H.-C. (2012). Using collaborative filtering to support college students' use of online forum for English learning. Computers and Education, 59(2), 628-637. https://doi.org/10.1016/j.compedu.2012.02.007

Wang, Z., Zhao, R., Xu, Y., Li, X., Zuo, M., y Ye, J. (2019). Interactive Discourse Analysis Based on the Forum Text Mining in Cloud Classroom. International fournal of Information and Education Technology, 9(3), 178-183. https://doi.org/10.18178/ijiet.2019.9.3.1195

Zhao, X., Jiang, Z., y Gray, J. (2019). Text classification and topic modeling for online discussion forums: An empirical study from the systems modeling community. En A. Fiori (Ed.), Trends and Applications of Text Summarization Techniques (pp. 151-186).

Zou, W., Hu, X., Pan, Z., Li, C., Cai, Y., y Liu, M. (2021). Exploring the relationship between social presence and learners' prestige in MOOC discussion forums using automated content analysis and social network analysis. Computers in Human Behavior, 115, 1-17. https://doi.org/10.1016/j.chb.2020.106582 